\title{
Influência de tratamento dentinário com EDTA sobre a resistência de união de sistemas adesivos autocondicionantes
}

\author{
Effectiveness of EDTA in restorative procedures with self-etching adhesives \\ Camila Cruz LORENZETTI a* (D), Marcela Caroline da Silva PEREIRA ${ }^{a}$ (D), Milton Carlos KUGA ${ }^{\mathrm{a}}$ (1), \\ José Roberto Cury SAAD ${ }^{\mathrm{a}}$ (1), Edson Alves de CAMPOS ${ }^{\mathrm{a}}$ (1) \\ aUNESP - Universidade Estadual Paulista, Faculdade de Odontologia de Araraquara, Departmento de Odontologia \\ Restauradora, Araraquara, SP, Brasil
}

\author{
Como citar: Lorenzetti CC, Pereira MCS, Kuga MC, Saad JRC, Campos EA. Influência de tratamento dentinário com EDTA \\ sobre a resistência de união de sistemas adesivos autocondicionantes. Rev Odontol UNESP. 2019;48:e20190007. \\ https://doi.org/10.1590/1807-2577.00719
}

\begin{abstract}
Resumo
Introdução: Os adesivos autocondicionantes apresentam técnica adesiva simplificada e diminuição na sensibilidade pós-operatória. Quando aplicados sobre a dentina, atuam sobre a smear layer, cuja efetiva remoção ainda constitui um desafio. Uma substância utilizada como pré-tratamento dentinário na ajuda da remoção da smear layer é o etilenodiamino tetracético dissódico (EDTA). Objetivo: Avaliar os efeitos do EDTA em associação com sistemas adesivos autocondicionantes na resistência de união ao microcisalhamento. Material e método: Foram seccionadas as superfícies oclusais de 72 terceiros molares humanos extraídos, para expor superfície dentinária plana. Os dentes preparados foram divididos em: dentina sem pré-tratamento; dentina tratada com EDTA 0,1M; dentina tratada com EDTA 0,5M. Cada grupo recebeu tratamento de três sistemas adesivos autocondicionantes: Single Bond Universal (SBU), AdheSE (AdheSE) e Clearfil SE Bond (CSEB). Foi delimitada a área adesiva e assim posicionada uma matriz transparente, para inserção com resina composta Z250XT seguida de polimerização por 40 segundos. Após 24 horas de armazenamento, os dentes foram submetidos ao ensaio de microcisalhamento. Os dados obtidos foram analisados estatisticamente, utilizando-se os testes Two-Way ANOVA e Bonferroni, e sendo considerado nível de significância de 5\%. Resultado: Não houve diferença estatística significante na resistência de união entre os pré-tratamentos dentinários $(\mathrm{p}=0,8353)$, porém houve diferença significativa entre os adesivos $(\mathrm{p}<0,05)$ CSEB e AdheSE, mostrando semelhança entre CSEB e SBU, e entre SBU e AdheSE. Conclusão: 0 pré-tratamento dentinário com solução de EDTA não afetou a resistência de união com nenhum dos sistemas adesivos autocondicionantes utilizados.
\end{abstract}

Descritores: Dentina; adesivo; EDTA.

\begin{abstract}
Introduction: Self-etching adhesives have simplified adhesive technique and decreased the postoperative sensitivity. When applied on dentin, it acts on the smear layer, but it is still a challenge in the effectiveness of this removal. A substance used as a pre-treatment for the removal of the smear layer is disodium ethylenediaminetetraacetic acid (EDTA). Objective: To evaluate the effects of EDTA in association with self-etching adhesive systems on micro-shear bond strength. Material and method: The occlusal surfaces of 72 extracted human third molars were sectioned to expose a flat dentin surface. The prepared teeth were divided into: dentin without pre-treatment; dentin treated with 0.1M EDTA; dentin treated with 0.5M EDTA. Each group received treatment of 3 self-etching adhesive systems: Single Bond Universal (SBU), AdheSE (AdheSE), and Clearfil SE Bond (CSEB). The adhesive area was delimited and positioned a transparent matrix for insertion with Z250XT composite resin followed by polymerization for 40 seconds. After $24 \mathrm{~h}$ of storage, the teeth were submitted to the micro-shear test. The data were analyzed statistically using the Two-Way ANOVA and Bonferroni tests, considering a level of significance of 5\%. Result: There was no statistically significant difference in bond strength between dentin pre-treatments $(p=0.8353)$,
\end{abstract}


but showed a significant difference between CSEB and AdheSE adhesives ( $p<0.05$ ), showing similarity between CSEB and SBU, and between SBU and AdheSE. Conclusion: Dentin pretreatment with EDTA solution did not affect bond strength with any of the self-etching adhesive systems used.

Descriptors: Dentin; adhesive; EDTA.

\section{INTRODUÇÃO}

A Odontologia contemporânea baseia-se em procedimentos minimamente invasivos, dependendo assim de uma adesão efetiva dos materiais restauradores. A adesão de materiais restauradores à dentina acontece pela infiltração e polimerização de monômeros hidrófilos, presentes no sistema adesivo e na malha de colágeno exposta pelo condicionamento ácido, dando origem a uma camada denominada camada híbrida ${ }^{1}$.

Os sistemas adesivos autocondicionantes são compostos por monômeros hidrófilos ácidos, água, HEMA (hidroxil etil metacrilato) e dimetacrilatos bifuncionais ${ }^{2}$. Estes sistemas adesivos têm como vantagens a simplificação da técnica adesiva e a diminuição da sensibilidade pósoperatória, assim como selamento marginal efetivo, tanto em margens de esmalte como em dentina ${ }^{3}$.

Assim que o sistema adesivo autocondicionante é aplicado sobre a dentina recém-preparada, este necessita ultrapassar a barreira de smear layer para então atuar sobre a dentina. Uma substância que pode ser utilizada para a remoção da smear layer é a solução de etilenodiamino tetracético dissódico (EDTA), o qual é derivado de um ácido fraco, capaz de promover a quelação de íons cálcio da dentina em pH alcalino. O EDTA atua sobre os componentes inorgânicos da smear layer, provocando desmineralização da dentina peri e intertubular ${ }^{4}$ sem alterar as proteínas dentinárias, evitando assim grandes alterações da estrutura fibrilar do colágeno presente na dentina ${ }^{5}$. Essa substância também apresenta ligação covalente aos íons metálicos e atrai íons de cálcio presentes na hidroxiapatita da dentina ${ }^{6}$. Outros autores consideram que o EDTA provoca a desnaturação das fibras colágenas ${ }^{7}$ e sua utilização previamente à aplicação de sistemas adesivos autocondicionantes poderia permitir uma interação dos monômeros com a estrutura dentária.

Em Cecchin et al. ${ }^{4}$, no qual avaliou-se a resistência de união à microtração de sistema adesivo autocondicionante aplicado à dentina após uso de substâncias químicas, o grupo que recebeu irrigação da dentina com hipoclorito de sódio apresentou maiores valores de resistência de união. Essa efetividade diminuiu quando foi aplicado seguido do uso de EDTA 17\%. Assim, é considerado de grande importância determinar se a utilização de substâncias condicionadoras da dentina seria útil, a fim de permitir a obtenção de um substrato mais adequado para a impregnação dos monômeros do sistema adesivo.

\section{OBJETIVO}

O objetivo deste trabalho foi avaliar os efeitos do EDTA em associação com sistemas adesivos autocondicionantes, observando seus efeitos sobre a resistência de união ao microcisalhamento.

\section{MATERIAL E MÉTODO}

Neste estudo, foram utilizados 72 terceiros molares humanos extraídos, isentos de cárie e mantidos em solução de timol $1 \%$ a $4^{\circ} \mathrm{C}$ até o momento de sua utilização. Este trabalho foi aprovado pela Comissão de Ética em Pesquisa (Parecer n.ํㅜ 2.966.013). 
A superfície oclusal de cada dente foi seccionada utilizando-se disco de diamante em rotação controlada e refrigeração com água (Isomet 1000, Buehler Ltd., Lake Bluff, IL, USA), com o objetivo de expor superfície dentinária plana. Foram utilizadas lixas com granulações 320 e 600, para padronizar a smear layer sobre estas superfícies. Cada dente foi incluído em um cilindro de PVC com $2 \mathrm{~cm}$ de altura e $1,5 \mathrm{~cm}$ de diâmetro, e preenchidos com resina acrílica autopolimerizável.

Os dentes preparados foram aleatoriamente divididos de acordo com o tipo de tratamento do substrato dentinário:

- Dentina sem tratamento prévio com EDTA: Os dentes não receberam tratamento prévio com EDTA, sendo aplicado apenas o sistema adesivo autocondicionante.

- Dentina condicionada com EDTA 0,1M: Foi aplicado sobre a dentina EDTA 0,1M, sendo utilizado sobre a superfície por um período de 60 segundos de forma passiva, seguido de lavagem abundante e secagem com papel absorvente.

- Dentina condicionada com EDTA 0,5M: Foi aplicado EDTA 0,5M sobre a dentina por um período de 30 segundos de forma passiva e, em seguida, realizada lavagem abundante e secagem com papel absorvente.

Após o tratamento do substrato, com os dentes aleatoriamente divididos de acordo com o tipo de tratamento dentinário, foram utilizados os sistemas adesivos Single Bond Universal - 3M ESPE (SBU), AdheSE - Ivoclar Vivadent (AdheSE) e Clearfil SE Bond - Kuraray Co. (CSEB), seguindo as recomendações de cada fabricante.

Para delimitar a área de adesão, foram feitos quatro orifícios em fita dupla-face, com auxílio de um perfurador de lençol de borracha, sendo a fita colocada sobre a superfície dentinária. Após o procedimento adesivo, a parte superior da fita foi removida e foram confeccionados os espécimes. Matrizes transparentes, de 0,7 $\mathrm{mm}$ de diâmetro interno e $1 \mathrm{~mm}$ de altura (Tygon tubing), foram cortadas com auxílio de uma lâmina de bisturi, para que a área de união fosse padronizada. As matrizes foram posicionadas sobre cada orifício e sua porção interna foi preenchida com resina composta Z250 na cor A3 (3M ESPE, St. Paul, MN, EUA), com polimerização durante 40 segundos. Os materiais foram polimerizados com a mesma unidade de luz LED (Bluephase, Ivoclar, Vivadent), com uma intensidade relativa de saída de pelo menos $1.200 \mathrm{~mW} / \mathrm{cm}^{2}$.

As matrizes foram removidas imediatamente com auxílio de uma lâmina de bisturi, para expor os cilindros de resina composta com área de união de $0,38 \mathrm{~mm}^{2}$. A fita dupla-face foi removida e os dentes foram armazenados em ambiente úmido por 24 horas, para posterior realização do teste de resistência de união ao microcisalhamento.

Após 24 horas de armazenamento, os dentes foram posicionados em um dispositivo para ensaio de resistência de união ao microcisalhamento acoplado à máquina de ensaios universal EMIC, com uma célula de carga de $500 \mathrm{kgF}$. 0 carregamento foi aplicado o mais próximo possível da interface de união na base dos cilindros, com o auxílio de uma alça de fio de aço com $0,25 \mathrm{~mm}$ de diâmetro. 0 carregamento foi realizado com velocidade de travessa de $0,5 \mathrm{~mm} / \mathrm{min}$ até a fratura dos espécimes.

A resistência de união ao microcisalhamento foi calculada dividindo-se a força máxima registrada durante o ensaio, em newtons, pela área de união, em $\mathrm{mm}^{2}$, e expressa em $\mathrm{MPa}$.

Os dados obtidos foram analisados utilizando Two-Way ANOVA e, após, o teste de Bonferroni, considerando nível de significância de 5\%. 


\section{RESULTADO}

Verificou-se que não houve diferença estatística significante na resistência de união entre os diferentes tratamentos dentinários aplicados ( $\mathrm{p}=0,8353)$, assim como também na interação do tratamento com os diferentes adesivos utilizados $(\mathrm{p}=0,2827)$ (Tabela 1). Porém, mostrou-se diferença significativa no fator adesivo $(\mathrm{p}<0,05)$. Na Tabela 2 , é possível verificar que houve diferença significante na resistência de união entre os adesivos Clearfil SE Bond (CSEB) e AdheSE, no grupo que não houve tratamento dentinário, mostrando semelhança entre os adesivos SBU e CSEB.

Tabela 1. Two-Way ANOVA para sistemas adesivos aplicados após diferentes tratamentos dentinários $(p<0,05)$

\begin{tabular}{cccccc}
\hline Fonte de variação & GL & SQ & QM & F & Valor-p \\
\hline Interação & 4 & 137,7 & 34,43 & 1,292 & 0,2827 \\
Adesivo & 2 & 284,9 & 142,4 & 5,345 & 0,0072 \\
Tratamento & 2 & 9,617 & 4,808 & 0,1804 & 0,8353 \\
Resíduo & 63 & 1679 & 26,65 & & \\
\hline
\end{tabular}

GL = Graus de Liberdade; $\mathrm{SQ}$ = Soma de Quadrados; QM = Quadrado Médio; F = Valor-F.

Tabela 2. Médias ( \pm desvio padrão) da resistência de união ao microcisalhamento (MPa) de sistemas adesivos aplicados após diferentes tratamentos dentinários (Two-Way ANOVA e Bonferroni, $p<0,05$ )

\begin{tabular}{cccc}
\hline & CSEB & SBU & AdheSE \\
\hline sem & $21,32( \pm 4,96) \mathrm{Aa}$ & $19,77( \pm 3,96) \mathrm{Aab}$ & $13,67( \pm 4,51) \mathrm{Ab}$ \\
EDTA 0,1M & $18,43( \pm 4,86) \mathrm{Aa}$ & $20,01( \pm 7,47) \mathrm{Aa}$ & $13,96( \pm 3,56) \mathrm{Aa}$ \\
EDTA 0,5M & $18,01( \pm 5,38) \mathrm{Aa}$ & $17,31( \pm 4,41) \mathrm{Aa}$ & $17,15( \pm 6,23) \mathrm{Aa}$ \\
\hline
\end{tabular}

CSEB = adesivo Clearfil SE Bond; SBU= adesivo Single Bond Universal; AdheSE = adesivo AdheSE.

Letras minúsculas iguais na mesma linha representam ausência de diferença estatística; letras maiúsculas iguais na mesma coluna representam ausência de diferença estatística $(\mathrm{p}<0,05)$.

\section{DISCUSSÃo}

Os sistemas adesivos autocondicionantes se apresentam como uma boa alternativa restauradora, devido à sua facilidade de utilização e à técnica menos sensível que os sistemas adesivos do tipo condiciona-e-lava. Essa abordagem autocondicionante elimina a fase de lavagem do condicionamento ácido, o que não diminui apenas o tempo de aplicação clínica, mas também reduz significantemente a sensibilidade técnica ou o risco de cometer erros durante a aplicação ${ }^{8}$.

Alguns fatores - como o tipo de substrato, o sistema adesivo utilizado (autocondicionante ou condiciona-e-lava) e a presença de umidade, contaminantes, agentes dessensibilizantes e adstringentes - podem influenciar a resistência de união ${ }^{9}$. Um dos fatores responsáveis pela degradação da camada híbrida e a perda do selamento dentinário, afetando assim a resistência de união dos adesivos com a dentina, são as metaloproteinases (MMPs), as quais são responsáveis pela degradação do colágeno ${ }^{10}$. Etilenodiamino tetracético dissódico (EDTA) é um quelante efetivo entre íons cálcio e zinco, atuando também na inativação da atividade enzimática das MMPs e na remoção da smear layer ${ }^{11}$, podendo inclusive ter um efeito desmineralizador igual ou até superior ao do ácido fosfórico ${ }^{12}$. A camada de smear layer pode ser benéfica, porque reduz a permeabilidade da dentina e impede ou atenua a penetração de bactérias nos túbulos dentinários. Porém, pode também ser prejudicial, pois atua como uma barreira para a penetração de soluções irrigadoras, medicamentos e monômeros adesivos no interior dos túbulos dentinários ${ }^{6}$. 
Os sistemas adesivos autocondicionantes com grau de acidez muito forte desmineralizam profundamente a dentina, o que pode favorecer o surgimento da degradação da camada híbrida, tornando essa integridade da interface adesiva fraca em longo tempo ${ }^{13}$. Os adesivos autocondicionantes podem ser de condicionamento ultrassuave $(\mathrm{pH}>2,5)$, suave $(\mathrm{pH}=2)$, intermediário forte $(1<\mathrm{pH}<2)$ e de acidez forte $(\mathrm{pH}<1)^{8}$. Com o $\mathrm{pH}$ mais alto dos adesivos autocondicionantes universais $(\mathrm{pH}>2,5)$ e assim um condicionamento ácido menor, é esperada uma união mais química entre os monômeros funcionais e a hidroxiapatita do que uma interação mecânica com o substrato ${ }^{14}$. Com essa propriedade, poderia se esperar uma menor adesão do adesivo SBU (passo único) quando comparado aos adesivos utilizados de dois passos, porém foi observado o contrário, o que é sugerido ser devido a outras propriedades.

Adesivos autocondicionantes de duas etapas, assim como o CSEB e o AdheSE utilizados neste estudo, apresentam em seu primer o monômero funcional 2-hidroxietil metacrilato (HEMA) e a água, que atuam na infiltração de monômeros resinosos no substrato, favorecendo a resistência de união ${ }^{8}$. Embora os autocondicionantes de uma etapa (universais) apresentem uma composição mais complexa com a mistura de monômeros hidrófilos e hidrofóbicos, e muitas vezes ausência do HEMA, o SBU apresenta esse monômero ${ }^{11}$, o que pode favorecer sua resistência de união e pode ter colaborado para seu semelhante desempenho, neste estudo, ao CSEB. Takamizawa et al. ${ }^{15}$ encontraram em seu estudo valores de resistência de união maior para os adesivos de duas etapas do que para os universais, única etapa.

Outro fator que influencia na resistência de união é a presença de um monômero chamado MDP, que se encontra na composição dos adesivos CSEB e SBU. Yoshida et al. ${ }^{16}$ demonstraram que este monômero é capaz de formar ligações iônicas fortes com o cálcio presente na dentina. Este monômero foi classificado como o mais promissor para a ligação química da hidroxiapatita do esmalte ou da dentina ${ }^{17}$. A presença deste monômero pode ser também responsável pelas maiores médias de resistência de união ao microcisalhamento e pela semelhança entre SBU e CSEB, assim como encontrado no estudo de Isolan et al. ${ }^{18}$, no qual mostrou-se semelhança na resistência de união entre esses dois adesivos aplicados também sobre a dentina. A ausência deste mesmo monômero no AdheSE pode ser relacionado com seus menores valores de resistência de união.

A avaliação da influência do pré-tratamento da dentina com EDTA em duas diferentes concentrações não mostrou diferença significativa do grupo que não recebeu esse tratamento, $o$ que difere do estudo de Luque-Martinez et al. ${ }^{19}$, que mostraram uma retenção significantemente maior no grupo tratado com EDTA em relação ao grupo controle, quando avaliaram performance clínica de restaurações cervicais por 18 meses, realizadas com sistemas adesivos autocondicionantes, devido a esta substância (EDTA) produzir uma dissolução seletiva da hidroxiapatita. Os mesmos autores ${ }^{19}$ sugerem que a preservação da interface dentina-resina com o pré tratamento dentinário com EDTA seja mais resultante do efeito de condicionamento suave do substrato do que pelo efeito inibidor de MMPs, embora o efeito de inibir a ação das MMPs na degradação da camada híbrida seja conhecido na literatura ${ }^{11}$. 0 fato de o EDTA desmineralizar superficialmente e de ter sido utilizado neste estudo de forma passiva pode ter influenciado o resultado semelhante ao grupo sem tratamento. Costa Lima et al. ${ }^{20}$ relataram que aplicação de forma ativa proporciona um aumento na remoção da smear layer, aumentando sua efetividade. Cecchin et al. ${ }^{4}$ também não encontraram diferença significativa entre o grupo que recebeu pré-tratamento dentinário com EDTA associado a $\mathrm{NaOCl}$ e o grupo controle (sem solução de irrigação), sugerindo que a erosão dentinária superficial causada pelo EDTA não afetou a união entre os monômeros resinosos e a dentina.

A mensuração da resistência de união é um dos métodos mais comuns para a avaliação das propriedades adesivas dos materiais restauradores. Vários métodos mecânicos - tais como tensão, flexão, ensaios de corte, cisalhamento e microtração - são usados para avaliar a união ao substrato dental ${ }^{21}$. O teste de microcisalhamento tem sido rotineiramente aplicado $^{22,23} \mathrm{e}$, 
diferentemente do teste de microtração, não há necessidade de se realizarem cortes dos espécimes, tornando-o mais simples e fácil de ser realizado ${ }^{8}$.

\section{CONCLUSÃO}

0 pré-tratamento dentinário com solução de EDTA não afetou a resistência de união com o uso de sistemas adesivos autocondicionantes. No grupo sem tratamento dentinário prévio ao uso dos sistemas adesivos, o adesivo SBU apresentou-se semelhante ao AdheSE e CSEB, sendo AdheSE e CSBE diferentes entre si.

\section{REFERÊNCIAS}

1. Brackett MG, Tay FR, Brackett WW, Dib A, Dipp FA, Mai S, et al. In vivo chlorhexidine stabilization of hybrid layers of an acetone-based dentin adhesive. Oper Dent. 2009 Jul-Aug;34(4):379-83. http://dx.doi.org/10.2341/08-103. PMid:19678441.

2. Tay FR, King NM, Chan KM, Pashley DH. How can nanoleakage occur in self-etching adhesive systems that demineralize and infiltrate simultaneously? J Adhes Dent. 2002;4(4):255-69. PMid:12666745.

3. Leinfelder KF, Kurdziolek SM. Self-etching bonding agents. Compend Contin Educ Dent. 2003 Jun;24(6):447-54.

4. Cecchin D, Farina AP, Galafassi D, Barbizam JV, Corona SA, Carlini-Júnior B. Influence of sodium hypochlorite and EDTA on the microtensile bond strength of a self-etching adhesive system. J Appl Oral Sci. 2010 Jul-Aug;18(4):385-9. http://dx.doi.org/10.1590/S1678-77572010000400011. PMid:20835574.

5. Habelitz S, Balooch M, Marshall SJ, Balooch G, Marshall GW Jr. In situ atomic force microscopy of partially demineralized human dentin collagen fibrils. J Struct Biol. 2002 Jun;138(3):227-36. http://dx.doi.org/10.1016/S1047-8477(02)00029-1. PMid:12217661.

6. Calt S, Serper A. Smear Layer removal by EGTA. J Endod. 2000 Aug;26(8):459-61. http://dx.doi.org/10.1097/00004770-200008000-00007. PMid:11199779.

7. Sauro S, Toledano M, Aguilera FS, Mannocci F, Pashley DH, Tay FR, et al. Resin-dentin bonds to EDTAtreated vs. acid-etched dentin using ethanol wet-bonding. Dent Mater. 2010 Apr;26(4):368-79. http://dx.doi.org/10.1016/j.dental.2009.12.008. PMid:20074787.

8. Van Meerbeek B, Yoshihara K, Yoshida Y, Mine A, De Munck J, Van Landuyt KL. State of the art of selfetch adhesives. Dent Mater. 2011 Jan;27(1):17-28. http://dx.doi.org/10.1016/j.dental.2010.10.023. PMid:21109301.

9. Powers JM, O'Keefe KL, Pinzon LM. Factors affecting in vitro bond strength of bonding agents to human dentin. Odontology. 2003 Sep;91(1):1-6. http://dx.doi.org/10.1007/s10266-003-0026-x. PMid:14505182.

10. Visse R, Nagase H. Matrix metalloproteinases and tissue inhibitors of metalloproteinases: structure, function, and biochemistry. Circ Res. 2003 May;92(8):827-39. http://dx.doi.org/10.1161/01.RES.0000070112.80711.3D. PMid:12730128.

11. Thompson JM, Agee K, Sidow SJ, McNally K, Lindsey K, Borke J, et al. Inhibition of endogenous dentin matrix metalloproteinases by ethylenediaminetetraacetic acid. J Endod. 2012 Jan;38(1):62-5. http://dx.doi.org/10.1016/j.joen.2011.09.005. PMid:22152622.

12. Jacques $P$, Hebling J. Effect of dentin conditioners on the microtensile bond strength of a conventional and a self-etching primer adhesive system. Dent Mater. 2005 Feb;21(2):103-9.

http://dx.doi.org/10.1016/j.dental.2003.12.004. PMid:15681008. 
13. De Munck J, Shirai K, Yoshida Y, Inoue S, Van Landuyt K, Lambrechts P, et al. Effect of water storage on the bonding effectiveness of 6 adhesives to Class I cavity dentin. Oper Dent. 2006 Jul-Aug;31(4):45665. http://dx.doi.org/10.2341/05-57. PMid:16924986.

14. Miyazaki M, Tsujimoto A, Tsubota K, Takamizawa T, Kurokawa H, Platt JA. Important compositional characteristics in the clinical use of adhesive systems. J Oral Sci. 2014 Mar;56(1):1-9. http://dx.doi.org/10.2334/josnusd.56.1. PMid:24739701.

15. Takamizawa T, Barkmeier WW, Sai K, Tsujimoto A, Imai A, Erickson RL, et al. Influence of different smear layers on bond durability of self-etch adhesives. Dent Mater. 2018 Feb;34(2):246-59. http://dx.doi.org/10.1016/j.dental.2017.11.002. PMid:29146046.

16. Yoshida Y, Nagakane K, Fukuda R, Nakayama Y, Okazaki M, Shintani H, et al. Comparative study on adhesive performance of functional monomers. J Dent Res. 2004 Jun;83(6):454-8. http://dx.doi.org/10.1177/154405910408300604. PMid:15153451.

17. Perdigão J, Ramos JC, Lambrechts P. In vitro interfacial relationship between human dentin and onebottle dental adhesives. Dent Mater. 1997 Jul;13(4):218-27. http://dx.doi.org/10.1016/S01095641(97)80032-2. PMid:11696900.

18. Isolan CP, Valente LL, Münchow EA, Basso GR, Pimentel AH, Schwantz JK, et al. Bond strength of a universal bonding agente and other contemporary dental adhesives applied on enamel, dentin, composite and porcelain. Appl Adhes Sci. 2014;2(25):1-10. http://dx.doi.org/10.1186/s40563-0140025-x.

19. Luque-Martinez I, Muñoz MA, Mena-Serrano A, Hass V, Reis A, Loguercio AD. Effect of EDTA conditioning on cervical restorations bonded with a self-etch adhesive: A randomized double-blind clinical trial. J Dent. 2015 Sep;43(9):1175-83. http://dx.doi.org/10.1016/j.jdent.2015.04.013. PMid:25963586.

20. Costa Lima GA, Aguiar CM, Camara AC, Alves LC, Dos Santos FA, do Nascimento AE. Comparison of smear layer removal using the Nd:YAG laser, ultrasound, ProTaper Universal system, and CanalBrush methods: an in vitro study. J Endod. 2015 Mar;41(3):400-4. http://dx.doi.org/10.1016/j.joen.2014.11.004. PMid:25600669.

21. Andrade AM, Garcia E, Moura SK, Reis A, Loguercio A, Silva LM, et al. Do the microshear test variables affect the bond strength values? Int J Dent. 2012;2012:618960. http://dx.doi.org/10.1155/2012/618960. PMid:23193406.

22. Yousry MM, ElNaga AA, Hafez RM, El-Badrawy W. Microshear bond strength and interfacial morphology of etch-and-rinse and self-etch adhesive systems to superficial and deep dentin. Quintessence Int. 2011 Oct;42(9):e96-106. PMid:21909495.

23. Mobarak EH, El-Badrawy WH. Microshear bond strength of self-etching adhesives to caries-affected dentin identified using the dye permeability test. J Adhes Dent. 2012 Jun;14(3):245-50. http://dx.doi.org/10.3290/j.jad.a22420. PMid:22282743.

\section{CONFLITOS DE INTERESSE}

Os autores declaram não haver conflitos de interesse.

\section{*AUTOR PARA CORRESPONDÊNCIA}

Camila Cruz Lorenzetti, UNESP - Universidade Estadual Paulista, Faculdade de Odontologia de Araraquara, Departamento de Odontologia Restauradora, Rua Humaitá, 1680, Centro, 14801903 Araraquara - SP, Brasil, e-mail: camilaclorenzetti@yahoo.com.br

Recebido: Janeiro 27, 2019

Aprovado: Janeiro 31, 2019 